\title{
Artículo
}

\section{Estudio de rentabilidad de la Biblioteca Pública Provincial de Valencia}

\author{
Por $\mathbf{M}^{\mathrm{a}}$ del Mar Amat de la Flor, Milagros Ortells Montón, Aurora Rapún Mombiela e \\ Ignacio Latorre Zacarés
}

\begin{abstract}
Resumen: Se esboza una estimación de cálculo de rentabilidad de las bibliotecas públicas, basándose en algunos modelos norteamericanos de análisis de costes-beneficios en bibliotecas públicas. Se toma como ejemplo la Biblioteca Pública Provincial de Valencia por reunir las condiciones necesarias para el estudio: nivel de uso elevado, público muy heterogéneo y poseer unos datos económicos y de uso fiables y bien conocidos. El objetivo es demostrar que invertir en bibliotecas públicas es informativa, formativa, cultural y socialmente muy rentable. La aplicación de modelos de análisis de costes-beneficios en bibliotecas públicas ayuda a evidenciar al ciudadano común y a la clase política la rentabilidad de la inversión en bibliotecas públicas y sirve para argumentar la necesidad de seguir incrementando la inversión en el servicio de lectura pública. Proseguir en España con unos indicadores al alza en el uso de las bibliotecas públicas debe suponer también un crecimiento de la inversión en el servicio.
\end{abstract}

Palabras clave: Análisis costes-beneficios, Cálculos de rentabilidad, Bibliotecas públicas, Presupuestos, Biblioteca Pública Provincial de Valencia, Beneficios, ROI.

\section{Title: Profitability study of the Provincial Public Library of Valencia}

Abstract: We have outlined an estimate of the profitability of public libraries based on several models used in the United States that measure cost versus profits in public libraries. The Provincial Public Library of Valencia was chosen because it meets the following conditions: a high level of use, a very heterogeneous public, and reliable economic and user data. Our aim is to show that investment in public libraries is highly profitable in terms of the information and training offered and from a cultural and social point of view. The use of analytical models that measure costs versus profits in public libraries can serve to raise awareness on the part of both citizens and politicians of the benefits of investing in public libraries as well as to highlight the need for investment in public lending libraries to continue to grow. In order to ensure growth in the use of public libraries in Spain, increased investment is clearly needed.

Keywords: Cost-profit analysis, Profitability estimates, Public libraries, Budgets, Valencia, Benefits, ROI.

Amat de la Flor, $M^{a}$ del Mar; Ortells Montón, Milagros; Rapún Mombiela, Aurora; Latorre Zacarés, Ignacio. «Estudio de rentabilidad de la biblioteca pública provincial de Valencia». En: El profesional de la información, 2006, septiembre-octubre, v. 15, n. 5, pp. 342-351. 


\section{Motivación del estudio y preguntas al aire}

La idea de realizar un cálculo sobre rentabilidad y bibliotecas públicas aplicado a nuestro entorno surge tras la lectura de los trabajos de Bruce T. Fraser, Timothy W. Nelson, Charles R. McClure y Jane B. Robbins ${ }^{1}$. En sus estudios se analizan los beneficios económicos directos e indirectos que aporta la biblioteca pública a la comunidad a la que sirve y se ofrecen métodos para mensurar el impacto económico que tiene sobre su ámbito de actuación. El cálculo se efectúa en ciertas bibliotecas americanas y, en este caso concreto, se aportaba el trabajo realizado sobre las bibliotecas públicas de Florida. De hecho, el Miami-Dade Public Library System publica en su web ${ }^{2}$ un informe anual sobre el balance de costos-beneficios de su sistema de lectura pública.

¿Se podría aplicar algún modelo de cálculo de rentabilidad sobre las bibliotecas públicas españolas?, ¿el saldo entre lo invertido por el contribuyente y lo recibido sería positivo?, ¿se cae en el mero reduccionismo económico por realizar cálculos de rentabilidad sobre un servicio que aporta sobre todo beneficios inmateriales?, ¿servirá un cálculo de la rentabilidad de las bibliotecas públicas españolas para que en ámbitos de decisión política se acabe con unos presupuestos que tienden a la escasez en muchos de los casos?

\section{¿Por qué el cálculo de rentabilidad económica sobre bienes y servicios culturales?}

Es opinión extendida que muchos servicios públicos no se pueden ni se deben analizar bajo una perspectiva económica, sino que producen un beneficio inmaterial, no cuantificable, como es el acceso progresivo a una mejor atención sanitaria, a una educación de calidad, a una extensión del conocimiento cultural, a un equilibrio igualitario entre capas sociales, etc. Son de carácter generalmente oneroso y que en pocos casos son atendidos desde la empresa privada pero que, sin embargo, son absolutamente necesarios para el progreso y bienestar de la sociedad. Las bibliotecas públicas españolas dependen casi en un $100 \%$ de la financiación pública y huelga decir la importancia que poseen para la formación, información y ocio de la comunidad. También es creencia general que son servicios que no se deben analizar sólo bajo una perspectiva de beneficios económicos y que han de ser costeados por medio de presupuestos públicos. Los autores de este artículo también estamos convencidos de ello y lejos de nuestras intenciones está someter el servicio de bi- blioteca pública, de evidentes beneficios culturales y sociales, al dictado puramente económico o al interés crematístico de la empresa privada. Pero entonces, ¿por qué realizar un cálculo de análisis de costes-beneficios sobre una biblioteca pública española?

Entre las motivaciones del estudio americano para realizar el cálculo de la rentabilidad de su sistema de lectura pública está la de informar al contribuyente de que su inversión en bibliotecas revierte de una manera muy positiva en la comunidad, todo ello en un contexto de cierta preocupación porque algunos de los indicadores de utilización de las bibliotecas en Norteamérica estaban a la baja (préstamos de documentos). Sin embargo, en España, los indicadores de utilización del sistema de lectura pública están en constante aumento, por lo que la motivación del estudio la orientamos hacia estos dos puntos:

-Evidenciar a la opinión pública que invertir en bibliotecas públicas produce unos beneficios directos $\mathrm{e}$ indirectos sobre la comunidad muy superiores a sus costos.

- Proporcionar argumentos para acabar con la escasez de dotación presupuestaria que muchas veces afecta al servicio de biblioteca pública, o para que en tiempos de crisis económica los recortes presupuestarios no empiecen por nuestros centros.

El método de análisis de costes-beneficios ayuda a demostrar que los beneficios derivados del servicio de biblioteca pública superan con mucho sus costes económicos. Generalmente, los artículos sobre centros de información y valor económico de la información se han aplicado sobre instituciones muy cercanas profesionalmente a las nuestras como los centros de documentación ${ }^{3}$ pero, en este caso, la voluntad era centrarse en el ámbito de las bibliotecas públicas.

\section{Bibliotecas públicas en España y presupuestos}

La consolidación del estado democrático y autonómico español ha supuesto un impulso decisivo sobre las bibliotecas públicas como queda demostrado en el estudio de Hilario Hernández Las bibliotecas públicas en España. Una realidad abierta centrado en la década de los 90 del s. $\mathrm{XX}^{4}$. La inversión en nuevos puntos de servicio de lectura pública (de 2.663 en el año 1990 a 4.661 en 2003) ha sido respondida con un incremento muy significativo de los indicadores de utilización de las bibliotecas públicas españolas: 16,4 millones de préstamos en 1990 frente a los 44 millones de 2003. Los datos del período 2001-2003 proporcio- 
nados por la Subdirección General de Coordinación Bibliotecaria del Ministerio de Cultura a través de la base de datos Alzira ${ }^{5}$ demuestran que el sistema bibliotecario público español sigue creciendo en sus principales parámetros de utilización (44 millones de préstamos y 77 millones de visitas en el año 2003 frente a 32 millones de préstamos y 58 millones de visitas en el 2001) y que la cobertura del servicio de biblioteca pública está cercana a su universalidad ( $97 \%$ de la población española).

Estas ratios positivas de crecimiento acelerado son debidas en parte a que el acceso universal a la cultura de biblioteca pública en España se ha desarrollado más tardíamente que en los países que nos sirven de referencia a nivel bibliotecario (Finlandia, Suecia, Dinamarca, Gran Bretaña, EUA, etc.). Así pues, a España aún le queda un margen de crecimiento grande, mientras otros modelos bibliotecarios mucho más evolucionados están preocupados por cierto estancamiento o incluso retroceso en sus cifras de utilización en los últimos años. La ratio de préstamo per cápita en España en el año 2003 era de 1,04 préstamos por habitante; muy distante de los 20 de Finlandia, los 13 de Dinamarca, 12 de Holanda, 10 de Canadá, 9 de Suecia, casi 7 de Gran Bretaña, 6 de EUA, etc. Estas diferencias estadísticas también se constatan en indicadores como el de población usuaria sobre población total, visitas per cápita, índice de rotación, etc.

\section{«El método de análisis de costes-beneficios ayuda a demostrar que los beneficios derivados del servicio de biblioteca pública superan con mucho sus costes económicos»}

Por lo que respecta a presupuestos parece que en España aún queda mucho por hacer en materia de inversión regular en bibliotecas públicas. El crecimiento en cuanto a la utilización a veces no parece traducirse en iguales proporciones en inversión en desarrollo bibliográfico (ver: Estudio sobre el desarrollo de las colecciones de las bibliotecas públicas en España ${ }^{6}$ ) lo que se traduce en unas colecciones aún muy por debajo de los parámetros europeos de dimensión, actualidad e inversión en recursos de información. Tampoco en muchos casos la plantilla de personal está suficientemente proporcionada ni con el nivel demográfico de la población a la que sirve, ni con las cifras de crecimiento de utilización del centro, ni con las prestaciones que se deben ofrecer desde una biblioteca pública.

Los gastos en bibliotecas públicas eran en el año 2003 de 348.703 .000 euros (gastos corrientes más los de inversión ${ }^{7}$ ) sumando los presupuestos de todas las administraciones públicas implicadas; aunque en el caso de la estatal, autonómica y de diputaciones ciertos gastos no se computen (personal técnico en las unidades de coordinación bibliotecaria, gastos centralizados de compra bibliográfica o de informática, campañas centralizadas de fomento de las bibliotecas y la lectura, etc.). En total, el indicador de gasto en España es de 8,1 euros/habitante, existiendo diferencias muy significativas entre comunidades autónomas (incidencias que están influyendo mucho en un servicio de biblioteca pública muy dispar según áreas geográficas).

A pesar de que en muchos casos es el centro cultural de mayor utilización en la comunidad local, los presupuestos reflejan ciertas deficiencias de inversión que pueden incidir en una ralentización del crecimiento. Además, contrastan muchas veces los presupuestos invertidos en bibliotecas públicas en compra bibliográfica frente, por ejemplo, a las universitarias donde existe una dotación presupuestaria mucho mayor ${ }^{8}$.

Quizás deberíamos incidir más en explicar que una mayor inversión en bibliotecas públicas se traduce rápidamente en una mayor calidad y dignidad del servicio que beneficia al contribuyente o convencer con argumentaciones sólidas de que invertir en bibliotecas públicas es cultural y socialmente muy rentable. Todo lo anteriormente dicho no es obstáculo para que en ciertas comunidades se realicen inversiones cuantiosas en la creación y renovación de infraestructuras bibliotecarias modélicas o que también en ciertas áreas geográficas la inversión en bibliotecas públicas vaya considerablemente al alza. Al respecto hay que señalar que el indicador de gasto por habitante también se encuentra en un alza constante, aunque se partiera de niveles muy bajos.

El gasto corriente en bibliotecas públicas en España es mantenido en un 1\% por el Estado, en un $28 \%$ por la administración autonómica y en un $69 \%$ por la local, que es la gran sostenedora financiera del sistema de lectura pública española. En el informe presentado por José Luís Zofío en el I Encuentro bibliotecas y municipios $^{9}$, se cuantificaba el gasto en bibliotecas (públicas y no públicas) en un 5,2\% del total de gasto público en cultura. Este valor supone que las bibliotecas están en el octavo escalafón de trece existentes del ranking de inversión en actividades culturales, por debajo de las deportivas, la música, juventud, patrimonio histórico, artes escénicas, artes plásticas y museos. Por otra parte, las cifras también indican que a diferencia de otros sectores culturales, el de las bibliotecas conlleva una utilización mucho más intensiva de mano de obra, pues casi el 16\% de los asalariados en cultura pertenecen al sector de bibliotecas. Estas magnitudes, $16 \%$ de asalariados para el 5,2\% de los gastos totales 
en cultura, implican que cerca del $51 \%$ de los gastos en bibliotecas públicas sean asumidos por el capítulo de personal (el 70\% si sólo contabilizamos los gastos corrientes, sin los de inversión). Es decir, queda muy poca liquidez para la inversión en el resto de capítulos: adquisición de fondos; mantenimiento de bibliotecas; inversiones en instalaciones, mobiliario o informática; creación de nuevos servicios; mercadotecnia; desarrollo de campañas de dinamización bibliotecaria; creación de nuevas bibliotecas, etc.

En la presentación del informe en las jornadas anteriormente aludidas, la utilización de métodos de cálculo macroeconómico sobre actividades no lucrativas, como las de las bibliotecas, tiende a infrarrepresentar su participación en la economía, pues la aportación de las bibliotecas y museos al Producto Interior Bruto sólo se valoraba en un 1 por 1.000. Sin embargo, la utilización de métodos y modelos como el empleado en este ensayo ayudan a visualizar claramente el impacto beneficioso que tiene en la sociedad el sistema de lectura pública valorando con precios de mercado los bienes y servicios ofrecidos.

\section{La Biblioteca Pública Provincial de Valencia (BPPV) ${ }^{10}$ y el motivo de su elección como centro representativo del estudio}

Está situada en un área céntrica de la capital, sirviendo por tanto a una población de 785.732 habitantes, aunque el área de influencia de la ciudad sea mucho mayor, tanto por su poblada periferia urbana como por ejercer de polo de atracción comercial, laboral y de oferta educativa y de ocio más allá de su propia provincia. Forma parte de la Red de Bibliotecas Públicas del Estado por lo que, mientras la titularidad del edificio y fondos pertenece al Estado, su gestión es asumida íntegramente por la Generalitat Valenciana.

El motivo de escogerla como ejemplo para ilustrar el presente estudio es su elevado nivel de utilización, así como la heterogeneidad de su público y los muy diferentes usos que hacen de ella sus usuarios: como centro de préstamo, de información, para consultar obras, para la lectura como ocio, como centro de consulta de internet y ordenadores multimedia, para obtener información legal, como lugar de estudio, para acudir a sus actividades de dinamización lectora y bibliotecaria, etc. Posee los niveles más altos de utilización de la Comunidad Valenciana y uno de los mayores de España. En el año 2004 se contabilizaron 522.222 visitas (una media de 1.800 por día efectivo de servicio) y 280.049 préstamos realizados (una media cercana a los 1.000 diarios por día efectivo de servicio). Su plantilla está compuesta por 39 funcionarios, 6 de ellos bibliotecarios profesionales. Su fondo

\section{Renovar (o comenzar) la sus- cripción a "El profesional de la información" es ágil y sencillo.}

\section{Usted puede gestionar online su suscripción conectándose a esta página web:}

http://www.elprofesionaldelainforma cion.com/suscripciones.html

\section{Si lo desea puede comunicar con nosotros dirigiéndose a esta di- rección de correo electrónico:} suscripciones@elprofesionaldelainforma cion.com

es de 177.307 documentos y está equipada con 63 ordenadores. Tiene su propia web y su catálogo está integrado en el Catálogo Colectivo de la Red Electrónica de Lectura Pública Valenciana. En cuanto a los costes, en el año 2004 ascendieron a 1.306.055 euros, aportando la Generalitat Valenciana el 95,4\% del total y el Estado el 4,5\%.

http://www.gva.es/bpv/

http://xlpv.cult.gva.es/

Un motivo esencial para su elección como modelo de análisis de costes-beneficios es que su presupuesto y gastos son bien conocidos y que sus datos estadísticos gozan de fiabilidad, ambos aspectos determinantes para realizar el estudio.

En la ciudad de Valencia existe otra unidad administrativa bibliotecaria, la Red Municipal de Bibliotecas, compuesta de una biblioteca central, 25 sucursales y una agencia de lectura. En total, en el año 2004 contabilizó 595.846 visitas y 158.485 préstamos. La coordinación entre la $B P P V$ y la Red Municipal es mínima por lo que no se puede hablar de un sistema único de lectura pública en la ciudad de Valencia. Así pues, el estudio se centra sólo sobre la unidad administrativa constituida por la $B P P V$.

\section{Los mimbres necesarios para realizar el estudio}

Para realizar un estudio de estas características es necesario contar con un conocimiento profundo de ciertos aspectos de la biblioteca o sistema bibliotecario 
a analizar. En primer lugar, el presupuesto y los gastos deben ser bien conocidos por el equipo de trabajo: volumen total de gastos, desagregación de los gastos según capítulos y participación en los gastos de las diferentes administraciones implicadas.

También se deben conocer los recursos o inputs con que se cuenta para poder ofrecer sus bienes y servicios: personal, infraestructura, edificio, colección bibliográfica y recursos de información en general, equipamiento de mobiliario e informático, etc. Además, para realizar un cálculo de beneficios es imprescindible tener bien contabilizados todos los outputs o productos de la biblioteca: préstamos realizados, consultas de información bibliográfica atendidas, sesiones de consulta pública de internet realizadas, consultas al opac y a la página web, ocupación de puestos de lectura, visitas diarias, participación en actividades de formación y de animación lectora, productos documentales realizados, etc.

\section{«Un buen servicio de biblioteca pública contribuye significativamente a la prosperidad cultural, formativa, informativa, social y económica de la comunidad a la que sirve»}

La puesta en valor de los productos y outputs de la biblioteca supone considerar los resultados a corto y medio plazo y, lo que es más importante, el impacto sobre la comunidad a la que se sirve que muchas veces exige un largo plazo para su evaluación. Si estos datos son relativamente fáciles de mensurar, sin embargo, es mucho más difícil cuantificar ciertos resultados e impactos beneficiosos que sobre la comunidad tienen las bibliotecas como es la mejora en la formación y educación, la elevación del nivel lector, la posesión de un mayor nivel informativo, el enriquecimiento del ocio activo, la satisfacción del usuario, el orgullo de poseer una buena biblioteca en la comunidad o el efecto de seguridad y buena acogida que produce el emplazamiento de una biblioteca sobre su entorno entre otros beneficios.

\section{Beneficios directos e indirectos del servicio de biblioteca pública}

Los servicios y usos proporcionados se traducen en una serie de beneficios para la comunidad:

-Directos: valor inmediato que tiene la biblioteca para todos los lectores que la usan o acceden a sus servicios.
-Indirectos: los obtienen terceros, o el conjunto de la comunidad, cuando la población la utiliza.

Un buen servicio de biblioteca pública contribuye significativamente a la prosperidad cultural, formativa, informativa y económica de la comunidad a la que sirve. Además, a pesar de que en general no se perciban los servicios y bienes ofrecidos como un beneficio económico, el equipo de estudio estadounidense en los trabajos anteriormente aludidos sí ha relacionado numerosos, variados y complejos beneficios económicos aportados por el sistema de lectura pública. Sin ánimo de relacionarlos todos, se pueden destacar los siguientes:

-Utilización de instalaciones por parte de asociaciones locales.

-Ahorros de los usuarios en costes de compra de libros y publicaciones periódicas y en compra o alquiler de material audiovisual.

-Ahorro en tarifas de conexión a internet, acceso a ancho de banda y en compra de ordenadores.

-Papel directo de la biblioteca como empleadora y también como consumidora de bienes y servicios. Las bibliotecas favorecen el mantenimiento de las librerías locales en estos tiempos de crisis para el sector.

Los puntos anteriores parecen contradecirse, pero la biblioteca ejerce un papel decisivo como creadora de lectores que además de utilizar los servicios gratuitos que les ofrece, se convierten en los clientes habituales de la industria del libro. En las sociedades lectoras se produce un circuito de retroalimentación donde se benefician las bibliotecas, las librerías, las editoriales y los autores. Por ejemplo, la biblioteca salva el posible primer escollo del coste a la gente joven y a la de bajo poder adquisitivo.

-Fomento del carácter democrático de la sociedad ofreciendo a toda la comunidad una información de calidad sin ningún tipo de discriminación.

- Mejora del nivel de lectura y de información de su comunidad y facilita la adopción de habilidades sociales y laborales.

-Fomento de la igualdad en el acceso a la información electrónica y mejora en el nivel de destreza informática de sus usuarios.

- Suministro de información muy útil a nivel personal: geográfica, sobre empleo, habilidades sociales y laborales, legal, consumo correcto, salud física y mental, ocio, etc.

—Facilita la integración de los inmigrantes en la comunidad. 


\begin{tabular}{|l|l|l|r|}
\hline Hemeroteca & Prensa & (Media anual) & 52,73 \\
\hline & Información legal & (Media por consulta) & 2,35 \\
\hline Sala de estudio & Puesto de estudio & (Media anual) & 85,56 \\
\hline & Consulta de documentos & (Media anual) & 72,08 \\
\hline Información y referencia & & (Media por consulta) & 3,45 \\
\hline Cuentacuentos & & (Media por sesión) & 5,21 \\
\hline
\end{tabular}

Tabla 1

-Apoyo a la educación: formal, no formal, autoformación, reciclaje profesional y a la "educación a lo largo de toda la vida" característica de la sociedad de la información.

-Buen lugar para el estudio.

-Enriquecimiento y mejora del ocio activo.

- Lugar seguro para los niños después del horario escolar y buen lugar de socialización.

-Proporciona seguridad a la zona donde se ubica y revaloriza su precio.

- Soporte para la cultura y arte locales.

-Motivo de orgullo para la comunidad.

- Mejora de la calidad de vida y del bienestar de sus usuarios.

\section{Cuantificar lo mensurable y lo no mensurable: métodos utilizados para calcular la rentabilidad del servicio de Ia BPPV}

Determinados beneficios directos para el usuario son fáciles de cuantificar económicamente, pues algunos bienes y servicios que disfrutan gratuitamente nuestros lectores en la biblioteca poseen un valor monetario real en el mercado: compra de libros y prensa, adquisición o alquiler de material audiovisual, utilización de sesiones de internet en cibercafés, acceso a bases de datos, etc.

Sin embargo, en otras ocasiones no poseen un valor monetario de mercado: disponibilidad de plazas de estudio, asistencia a la hora del cuento, servicio de información bibliográfica, acceso a una buena colección de recursos de información, etc. Pero, también existen fórmulas para calcular el valor de este tipo de bienes y servicios por medio de las propias estimaciones de nuestros usuarios que asignan un valor económico a los servicios y bienes que disfrutan. En este caso se recurre a encuestas y sondeos donde al usuario se le enfrenta ante una situación hipotética clara de tener que pagar por un servicio o bien renunciar a él. Deberá elegir si pagaría por el servicio y cuánto pagaría o si bien renunciaría a él. La asignación económica que el usuario pagaría nos sirve para realizar un cálculo razonable y estimado de lo que costaría con valor de mercado.
Pero es aún más complejo, y en algunos casos imposible, cuantificar con un valor monetario algunos de los beneficios indirectos que proporciona la biblioteca a su comunidad: ¿cómo calcular la satisfacción emocional del usuario por ser miembro de una buena biblioteca?, ¿es posible evaluar económicamente la sensación de seguridad que proporciona a la zona donde se ubica (aspecto importante en la $B P P V$ por su enclave actual frente a un barrio muy degradado que está en pleno proceso de reforma)?, ¿cómo valorar en dinero la mejora en los aspectos de formación e información que produce sobre la comunidad?, ¿quién puede calcular el beneficio que producen sobre los inmigrantes los recursos y servicios ofrecidos gratuitamente por nuestras bibliotecas?, ¿qué valor económico se asigna a la mejora en el bienestar y calidad de vida del ciudadano? Todos estos casos y algunos más quedan fuera del estudio realizado.

\section{«Cerca del $51 \%$ de los gastos en bibliotecas públicas son asumidos por el capítulo de personal»}

En la actualidad existen varios modelos para el cálculo de la rentabilidad en bibliotecas públicas que nos aportan una estimación más o menos aproximada. El análisis de costes-beneficios aplicado en el modelo norteamericano en sus diferentes variantes es un método fácil de entender por los usuarios y contribuyentes, por tanto, fácil de comunicar a la sociedad, aportando una visibilidad clara de los beneficios económicos que reporta la biblioteca a sus usuarios y a la comunidad en general. En el caso del Sistema de Lectura Pública de Miami-Dade se basa en el usual concepto ROI (return-on-investment) que se deduce tras calcular los beneficios totales directos aportados por el sistema a sus lectores y restarles la inversión realizada por los contribuyentes (ver nota 2). El saldo positivo obtenido demuestra al contribuyente que recibe por el servicio más de lo que paga por él.

En el caso de la $B P P V$ se ha optado por dos modelos de análisis: 
1. Modelo tipo Sistema de Lectura Pública Miami-Dade o qué es lo que se ahorra el usuario por utilizar gratuitamente los bienes y servicios de su biblioteca pública

En este caso, cuando los bienes y servicios se venden en el mercado (libros, prensa, material audiovisual, conexión a internet) se les asigna como valor de cálculo su precio real medio. Es decir, se le comunica al usuario cuál es el precio real que hubiera tenido que pagar si hubiera adquirido todos los documentos que ha utilizado en su biblioteca o pagado todos sus servicios a precio real de mercado. En el año 2003-2004, la Miami-Dade aplicó un valor medio por libro y material audiovisual prestado de 25 US\$ y de 10 cuando el material sólo era consultado como referencia. En el caso de la $B P P V$ se ha usado como valor medio por documento prestado el de 16,10 euros, resultado de hacer una media entre 75 precios de libros y material seleccionados $^{11}$. A diferencia del modelo de Miami, no existen datos del número de documentos utilizados en sala, por lo que no se ha cuantificado económicamente la consulta de documentos. El uso de internet se ha calculado al precio real de una sesión de uso en un cibercafé: 1 euro la hora.

\section{«En las sociedades lectoras se produce un circuito de retroalimentación donde se benefician las bibliotecas, las librerías, las editoriales y los autores»}

Cuando determinados servicios no disponen de un valor de mercado, se le ha preguntado a los usuarios por el valor económico que les asignarían para acceder a su uso: hemeroteca, asistencia a cuentacuentos, servicio de información bibliográfica, uso de la biblioteca como lugar de estudio, etc. En la tabla 1 se ofrecen los valores medios económicos asignados por los usuarios.

Estos resultados han servido como orientación para el estudio y han confirmado que los usuarios sí son conscientes del valor de los servicios proporcionados por la biblioteca, pero no se ha trabajado con estas cantidades aportadas por los encuestados ya que no se cuantifica el uso mensual o anual desagregado de estos servicios, con lo que el cruce de datos no es posible.

Finalmente se le pedía al usuario que indicara la cantidad que estaría dispuesto a pagar al año por el servicio general de la biblioteca (sin desagregar servicios). La media ponderada de todas las cantidades proporcionadas por los encuestados nos daría que el usua- rio de la $B P P V$ pagaría 147,71 euros al año por ser socio de la biblioteca y utilizar cualquiera de sus servicios. Esta cantidad nos sirve para cruzarla con el número de socios activos con carnet de la biblioteca ${ }^{12}$.

La suma de todos estos valores económicos nos proporciona los beneficios totales obtenidos por los usuarios de la biblioteca pública o lo que hubieran tenido que haber pagado en el mercado real por la compra o uso de todos los bienes y servicios utilizados gratuitamente. A estos beneficios totales hay que restarles los costes totales de la biblioteca. El resultado será el ROI o el montante económico recibido por la comunidad, que supera lo pagado por el contribuyente ${ }^{13}$.

\section{Modelo de cálculo propio}

Es muy parecido al anterior, pero en este caso el préstamo de libros y material audiovisual se calcula al mismo valor que cuesta alquilar un vídeo o dvd en las tiendas especializadas. Se ha utilizado como valor medio por documento prestado el de 3 euros, resultado de hacer una media entre los precios de alquiler de vídeos, dvds, videojuegos en varios establecimientos que proporcionan este servicio. En cuanto a los libros, se le ha asignado el mismo valor económico que a los documentos audiovisuales por no existir en el mercado un servicio equiparable de alquiler de libros. El precio estipulado para una hora de internet a partir de una media hecha entre varios cibercafés consultados es, como se dijo antes, de 1 euro/hora. El resto de ítems se sigue calculando en base al valor que según las encuestas realizadas pagaría el lector por disfrutar del bien o servicio.

En todo caso, ambos modelos aportan sólo una estimación razonable sobre el cálculo de beneficios ya que algunos no son computados, entre ellos casi todos los indirectos y algunos de los directos cuya cuantificación es bastante dificultosa. Además, este estudio es sólo un intento de aproximación al cálculo de rentabilidad sobre bibliotecas públicas españolas sin pretender un trabajo detallado que conllevaría sondeos complementarios sobre satisfacción del usuario, su opinión respecto a los diferentes impactos beneficiosos de la biblioteca pública, etc. Sin embargo, estos trabajos parciales ya demuestran claramente cómo los beneficios económicos de las bibliotecas públicas también en España superan a sus costes.

\section{Encuestas a usuarios en la BPPV}

Se elaboraron con el objetivo de asignar un valor económico a aquellos servicios que ofrece la biblioteca y que no tienen un valor monetario en el mercado, tales como disponibilidad de sitio de estudio, asistencia a la hora del cuento, servicio de información bibliográfica, acceso a una buena colección de recursos de información, hemeroteca, etc. Al usuario se le pre- 


\begin{tabular}{|l|c|c|c|}
\hline \multicolumn{1}{|c|}{2004} & $\begin{array}{c}\text { Generalitat } \\
\text { Valenciana }\end{array}$ & $\begin{array}{c}\text { Ministerio de } \\
\text { Cultura }\end{array}$ & \multicolumn{1}{c|}{ Total } \\
\hline Adquisiciones & $66.804,24$ & & $66.804,24$ \\
\hline Personal & $873.401,83$ & & $873.401,83$ \\
\hline Actividades & $12.775,89$ & & $12.775,89$ \\
\hline Mantenimiento & $241.722,50$ & & $241.722,50$ \\
\hline Otros gastos de inversión & $52.252,84$ & $59.098,13$ & $111.350,97$ \\
\hline Total & $1.246 .957,30$ & $59.098,13$ & $1.306 .055,43$ \\
\hline & $95,4 \%$ & $4,5 \%$ & \\
\hline
\end{tabular}

Tabla 2

\begin{tabular}{|l|r|l|r|}
\hline 2004 & & & \\
\hline Documentos prestados (libros, dvds, cds) & 262.431 & x 16,10 euros & $4.225 .139,10$ \\
\hline Usuarios de internet & 44.520 & x 1 euro & $44.520,00$ \\
\hline Socios & 85.673 & x 147,71 euros & $12.654 .758,83$ \\
\hline Total & & & $16.924 .417,93$ \\
\hline
\end{tabular}

\begin{tabular}{|l|r|}
\hline Total ingresos & $16.924 .417,93$ \\
\hline Total gastos & $1.306 .055,43$ \\
\hline Total beneficios & $15.618 .362,50$ \\
\hline
\end{tabular}

\begin{tabular}{|l|c|l|r|}
\hline 2004 & & & \\
\hline Documentos prestados (libros, dvds, cds) & 262.431 & $\times 3$ euros & $787.293,00$ \\
\hline Usuarios de internet & 44.520 & $\times 1$ euros & $44.520,00$ \\
\hline Socios & 85.673 & $\times 147,71$ euros & $12.654 .758,83$ \\
\hline Total & & & $13.486 .571,83$ \\
\hline
\end{tabular}

\begin{tabular}{|l|r|}
\hline Total ingresos & $13.486 .571,83$ \\
\hline Total gastos & $1.306 .055,43$ \\
\hline Total beneficios & $12.180 .516,40$ \\
\hline
\end{tabular}

Tabla 4

sentaba ante una situación hipotética clara de tener que elegir entre pagar una cierta cantidad por seguir disfrutando de un servicio ("Predisposición a pagar") o bien renunciar a él ("Predisposición a renunciar").

Se elaboró un modelo de encuesta que constaba de dos partes:

-Información general sobre los usuarios: edad, sexo, nivel educativo, lugar de procedencia y utilización por parte del usuario de otras bibliotecas.

- Servicios bibliotecarios que tiene que valorar el usuario en la encuesta. En esta parte se tuvo en cuenta la frecuencia de uso de cada uno de ellos y la cantidad que cada lector estaría dispuesto a pagar por cada uno. Para ello se optó por establecer una serie de rangos económicos con el fin de orientar al usuario, aunque también se ofreció la opción de respuesta abierta.
Asimismo, se diseñó una aplicación informática estadística ${ }^{14}$ para la tabulación y explotación de los datos.

Se cumplimentaron 119 encuestas entre usuarios de las diferentes secciones y servicios de la $B P P V$ : préstamo, hemeroteca, salas de ciencias y letras, infantil, hora del cuento, consulta pública de internet y multimedia. El primer dato positivo fue comprobar como un $89 \%$ de los encuestados tenían una predisposición a pagar por el servicio, frente a un $11 \%$ que ante la opción de pagar o tener que renunciar a él, eligió la renuncia.

\section{Resultados}

El total de costes desagregado por conceptos y administración financiadora de la BPPV en el año 2004 fue el mostrado en la tabla 2. Por lo tanto, 1.306.055 
euros es lo que el contribuyente pagó en el año 2004 por el mantenimiento e inversión en los servicios de la biblioteca pública.

\section{Beneficios según modelo Miami}

Se realiza el cálculo con los documentos prestados por su valor medio de compra; los usuarios de internet por el coste de conexión de 1 hora en el mercado y el número de socios inscritos que tuvo la $B P P V$ por el precio medio que los usuarios han asignado al servicio general (tabla 3).

\section{Beneficios según modelo de cálculo propio}

Se realiza el cálculo con los documentos prestados por su valor medio de alquiler en el mercado; los usuarios de internet por el coste de conexión de una hora en el mercado y el número de socios inscritos que tuvo la $B P P V$ por el precio medio que los usuarios han asignado al uso del servicio general (tabla 4).

\section{Conclusiones}

- Las bibliotecas públicas contribuyen a la prosperidad y desarrollo de la comunidad a la que sirven y se constituyen, en muchos casos, en el principal centro de información de la localidad y el centro cultural más utilizado.

- La ciudadanía es consciente del elevado valor positivo de poseer un servicio de lectura pública digno en su área o zona y reclama su instalación allí donde no exista el servicio de lectura pública o donde no se desarrolle en buenas condiciones.

- La biblioteca tiene que seguir siendo un servicio público, universal y gratuito, mantenido por presupuestos públicos y que continúe aportando una mejora en el bienestar y calidad de vida del ciudadano común.

- Los beneficios informativos, formativos, de ocio activo y culturales proporcionados por el uso y disfrute del sistema de lectura pública suponen un provecho económico directo superior a lo que el contribuyente paga por él. Así pues, invertir en bibliotecas públicas es informativa, formativa, cultural y socialmente muy rentable.

\section{Versión online de EPI}

Existe una versión electrónica de El profesional de la información, de uso gratuito para todos los suscriptores de la revista, que pueden acceder a través de internet a los textos completos y materiales gráficos publicados desde el año 2000.

\section{Más información en:}

http://www.elprofesionaldelainformacion.com/contenidos.html
-Determinados beneficios indirectos proporcionados por la biblioteca pública suponen beneficios inmateriales imposibles de calcular económicamente.

- Proseguir en España con unos indicadores al alza en el uso de las bibliotecas públicas pasa también por un crecimiento de la inversión en el servicio que, a su vez, se traduce rápidamente en una mejora en la calidad de la lectura pública que revierte en el contribuyente.

- La aplicación de modelos de análisis de costes-beneficios en bibliotecas públicas ayuda a evidenciar al ciudadano común y a la clase política la rentabilidad de la inversión en bibliotecas públicas.

—Los resultados de los estudios sobre rentabilidad sirven para argumentar la necesidad de seguir incrementando la inversión en el servicio de lectura pública y para que, además, en tiempos de crisis económica, las bibliotecas no sufran los "frecuentes" recortes presupuestarios.

-Aunque su uso supone un beneficio directo para el usuario en concepto de ahorro en costes de compra de libros y otros materiales, en realidad esto no afecta a la industria del libro, porque a la vez se está ayudando a crear una clase lectora y consumidora de productos culturales configurando un circuito de retroalimentación que beneficia a todo el sector. Donde son más potentes las bibliotecas públicas es más fuerte la industria y comercio del libro (librerías, editoriales, autores, etc.).

- Tras realizar los cálculos cuantitativos, sería conveniente añadir indicadores cualitativos del servicio de la biblioteca: actualidad de la colección, rapidez en la resolución de preguntas, confortabilidad de las instalaciones, modernidad y pertinencia del equipamiento informático, calidad de conexiones, calidad en la atención personalizada, etc.

\section{Notas}

1. McClure, Charles R.; Fraser, Bruce T.; Nelson, Timothy; Robbins, Jane B. "Descripción del impacto y los beneficios económicos de las bibliotecas públicas de Florida: resultados y aplicaciones metodológicas para trabajos futuros". En: Boletín de la Asociación Andaluza de Bibliotecarios, 2003, n. 72, pp. 73-101. Es una traducción de Antonio Lozano-Palacios y Encarnación Poyatos-Huertas del artículo original publicado en inglés:

McClure, Charles R.; Fraser, Bruce T.; Nelson, Timothy; Robbins, Jane B. Economic benefits and impacts from public libraries in the state of Florida. En: Library \& information science research, 2004, v. 24, n. 3, pp. 211-233. Consultado en: 15-11-05.

http://dlis.dos.state.fl.us/bld/finalreport/

2. Miami-Dade Public Library System. A report to our community 2003-2004. Consultado en 14-10-05.

http://www.mdpls.org/info/pdf/annual99-00.pdf

3. Latorre Zacarés, Jesús. "Cobrar por la información por qué no?”. En: Compactus: revista d'arxius i biblioteques, 2003, maig-agost, n. 7, pp. 24-26. Se analiza el volumen de negocio de los departamentos de docu- 
mentación de centros de innovación tecnológica y en su final resalta las afinidades que puede tener el modelo con centros de información "hermanos" como las bibliotecas públicas.

4. Hernández, Hilario. "Las bibliotecas públicas en España. Una realidad abierta". En: Las bibliotecas públicas en España. Una realidad abierta. Peñaranda de Bracamonte: Fundación Germán Sánchez Ruipérez, 2001, pp. 13-133. ISBN 84-89384-33-9.

5. Web de la base de datos estadística Alzira. Este programa ha sido posible gracias al exitoso trabajo cooperativo realizado por todas las administraciones públicas que trabajan en bibliotecas públicas a través del Grupo de Estadísticas de Bibliotecas Públicas.

http://travesia.mcu.es/cabotaje.asp

6. Estudio sobre el desarrollo de las colecciones de las bibliotecas públicas en España. Hilario Hernández (dir). Peñaranda de Bracamonte: Fundación

Germán Sánchez Ruipérez, 2002.

Consultado en 02-12-05.

http://www.bibliotecaspublicas.info/

7. Datos extraídos de la base de datos Alzira del $\mathrm{Mi}$ nisterio de Cultura.

http://travesia.mcu.es/cabotaje.asp

8. Mientras en el año 2001 la Red de Bibliotecas Universitarias y Científicas Españolas invertía 93.000.000 euros en compra de fondos bibliográficos, en el mismo año entre más de 4.000 centros de lectura pública se invertían 27.000.000 euros. Es paradójico -en el área estudiada en este artículo- que mientras en toda la Comunidad Valenciana con 532 centros de lectura pública se invertían en el año 2003 3.757.000 euros en compra de fondos bibliográficos, la Universitat de València (1 de las 6 universidades presentes en la Comunidad Valenciana) había invertido dos años antes 2.757.000 euros ella sola.

9. Zofío, José Luis. "La relevancia económica de la provisión de servicios culturales por las administraciones públicas: las bibliotecas". En: I Encuentro bibliotecas y municipio. La administración local y las bibliotecas en la democracia: Peñaranda de Bracamonte, 2005. Madrid: Ministerio de Cultura, 2005. Consultado en: 03-12-05.

http://travesia.mcu.es/

10. Los autores del artículo agradecemos la colaboración prestada por la dirección y la plantilla de la $B P P V$. Así mismo les felicitamos por su esfuerzo diario para ofrecer a la ciudad de Valencia y área de influencia un servicio de lectura pública digno.

11. Sobre una lista de 25 libros la media es de 16,33 euros, sobre una lista de $25 \mathrm{dvds}$ la media es de 15,67 euros y sobre una lista de 25 cds la media es de 16,32 euros. Entre los tres la media es de 16,10 euros.

12. En el año 2004 fueron 85.673 los socios con carnet de la biblioteca que lo utilizaron para alguna transacción de préstamo. Sin embargo, la creación del carnet único de usuario de la Red de Electrónica de Lectura Pública Valenciana provoca alguna distorsión, como que no se puedan computar como socios activos de la biblioteca aquellos usuarios con carnet de socio expedido en otras bibliotecas de la red que utilizan los servicios de la biblioteca. Este es un caso muy frecuente en la $B P P V$ debido especialmente a estudiantes de otras poblaciones que entre semana están en Valencia. También, no se registra como socios activos de la biblioteca a aquellos usuarios que sí la utilizan pero no poseen el carnet por

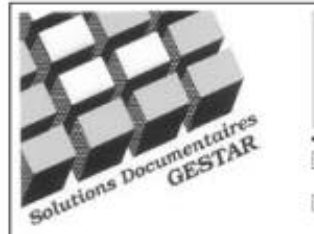

\section{Programa e-Documentik $\boldsymbol{c}_{B}$ $100 \%$ WEB}

\section{Las necesidades...}

Está buscando un programa informático de gestión documental a la vez fácil a utilizar, completo y potente para la gestión integrada de sus documentos sobre papel y de sus documentos electrónicos.

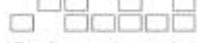

Quiere una herramienta que permite la gestión e indización de todos los tipos de documentos: informes y actas, actas notariales, fotografías, reglamentos, catálogos, ficheros sonoros, documentación de referencia,...

Desea adquirir o alquilar una solución $100 \%$ Web instalada en su servidor SQL o hacerla albergarse en nuestros servidores, sin límite del número de usuarios y que permite la gestión de varias bases de datos en la misma aplicación.

\section{Privilegia un producto concebido, desarrollado y soportado por} especialistas de la gestión documental que están en condiciones de guiarle para una integración armoniosa de esta herramienta informática.

\section{El programa e-Documentik ${ }^{\circledR}$ ..sistema integrado de gestión electrónica de los documentos analógicos y de los} documentos electrónicos.

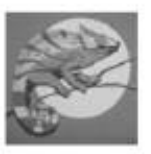
...la Solución para una gestión documental en nuestra era digital.

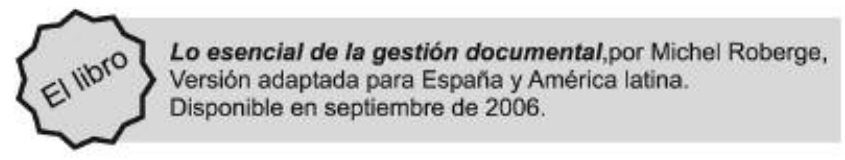

Solutions Documentaires GESTAR Av. Gran via de les Corts Catalanes, $780, \mathrm{At}^{\mathrm{t}} 2^{\mathrm{a}}$ 08013 Barcelona TEL:: (+34) 639617815

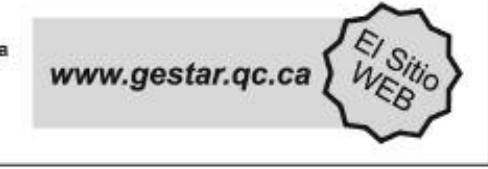

utilizar servicios que no requieren su posesión (consulta de documentos o de hemeroteca, utilización de un lugar de estudio, asistencia a actividades, etc.). Por lo tanto la cifra de socios activos de la biblioteca se podría revisar al alza.

13. Debido a las especiales características de la $B P P V$ no se han restado posibles costes de amortización de edificio. La biblioteca se ubica en un edificio histórico (el primer manicomio europeo) y como tal biblioteca fue inaugurada en 1979. Desde su apertura las intervenciones en el edificio han sido más bien de mantenimiento, aparte de alguna modesta ampliación. Así pues, el coste de la obra originaria del edificio se considera más que amortizado. Por otra parte, a los gastos de inversión anual no se les detrae los gastos de amortización de ordenadores, mobiliario, etc., que tendrán en los años de vida de estos equipos, ya que una biblioteca precisa de gastos de inversión continuos en tecnología y mobiliario.

14. Agradecemos a Javier Belmonte, técnico informático que presta sus servicios en la $D i$ rección General del Libro y Bibliotecas de la Generalitat Valenciana, su diseño de la aplicación informática.

\section{$M^{a}$ del Mar Amat de la Flor, técnica de bibliotecas. amat_marflo@gva.es}

Milagros Ortells Montón, técnica de bibliotecas de la Dirección General del Libro y Bibliotecas de la Generalitat Valenciana.

ortells_mil@gva.es

Aurora Rapún Mombiela, técnica de bibliotecas.

aurorarapun@yahoo.es

Ignacio Latorre Zacarés, técnico de archivos y bibliotecas de la Generalitat Valenciana.

latorre_ign@gva.es 